\title{
SURVEY OFOPTIMIZATION TECHNIQUES IN GESTURE RECOGNITION
}

\author{
Sharda.A.Chhabria ${ }^{1}$, R.V.Daraskar ${ }^{2}$, V.M.Thakare ${ }^{3}$ \\ ${ }^{I}$ Assistant Professor, IT, G.H.Raisoni College of Engg., Nagpur, India \\ ${ }^{2}$ Former Director, Disha Education Society,(DIMAT- Disha Technical Campus), Raipur, India \\ ${ }^{3}$ Professor \& Head,CSE, Amravati University, India
}

\begin{abstract}
In recent days significant interest is developed in gesture recognition domain because of need of different possible applications, that includes: search and rescue, hospital care, etc.

This paper recognizes need of parameter optimization for gesture recognition. Various optimization techniques are discussed like Convex Optimization, Contextual Video-Text Fusion, Optimized Video Densely Sampling, Gaussian Mixture Markov Random Field, Visual Tracking by Weighted Likelihood Maximization, Mean Variance Mapping Optimization
\end{abstract}

Keywords — component; Gesture Recognition; optimization; Gaussian mixture Model

\section{INTRODUCTION TO OPTIMIZATION}

\subsection{System with Optimization}

The task which makes a machine or layout as powerful or functional as possible is known as optimization or in which overall performance is maximized and error is minimized, can be called as optimised. An example is specification of constrained optimization problem for achievement of this state.

Error is minimized in the estimation process in optimum system.Parameters such as accuracy and reliability fincreases, if there is reduction in error in the estimation process. The goal is to obtain fast and quality performance in terms of time space and accuracy for the estimation process, by reducing number of parameter during process of fusion.

Iterative assessment of the fusion module performance is required, to solve constrained optimization problem of the system.Response Time is generally equated by performance and is measured in terms of throughput. The number of transactions processed in unit interval of time measurement gives throughput.Zero performance is given by non functioning system.

\subsection{System without Optimization}

The system's goal is only to decrease the price of compilation and to obtain the desiredoutput in systems without using optimization. In this approach suppose if there is breakpoint among statements of program and the program gets stoppedthen the recent value is assigned to any variable and user gets accurate results which is expected from the source code, that is statements are independent. Comparatively the price of compilation time and possibly the ability to debug the program will be decreased and performance is thus improved .

\section{OPTIMIZATION TECHNIQUES}

\subsection{Convex Optimization}

In a fusion problem that is distributed, cross-covariance's is initially assessed.As cross-covariance matricesof assessedvariables are not known and to combine the estimates linearly, the weighting coefficients are calculated. Two methods are employed, in the first one, priors are not used by the covariance matrices of the model and in second one, priors are used and the Bayesian machinery is engaged. Exploit the maximum-entropy principle for the former, in finding the optimal cross-covariance estimate and Wishart distributions is employed as priors for the latter, and maximum posteriori estimate is searched. Both problems need convex optimization for which solution can be obtained by current surviving techniques.Easy computation of weighting coefficients can be done after producing crosscovariance to make fusion occur.

\subsection{Contextual Video-Text Fusion}

The measure of appearance and behavior similarity are jointly optimized to generate probabilistic relationship among entities described in text and obtained in video data. Multiple assumptions, are maintained due to which ambiguity is managed that results from features that are not coinciding (conflicting) in the sources. Activity recognition scores has increased in this method of soft fusion above both individual source processing and non-probabilistic (hard) fusion in which synthetic data that have very less coinciding features among sources. Hard fusion outperforms single source and soft fusion when sources have over $60 \%$ coinciding features. To select the correct fusion algorithm to 
result in the highest activity recognition results and to determine whether soft or hard fusion is suitable for a dataset, this method is adaptable.

\subsection{Weighted Likelihood Maximization For Visual}

\section{Tracking [2]}

The recent mobile displays have developed a demand for an effective technique to interact with the information displayed in the HMD. In situation where the hands are required for other tasks, equipment's, which are controlled by hand such as keyboard or mouse, become clumsy. Gaze interaction along with the HMD can possiblygive a handsfree pointing technique. Peoplemay be benefit from an HMD with gaze control if they are usingaugmented and alternative communication tools.Daily activities, like wheelchair driving, would not be interrupted when communicating. People who do not have control of their hands could communicate on-the-move and in bed without any need of outside helpfor reposition of the equipment.

This algorithm is based on probabilistic real time tracking Gaussian mixture model (GMM) describes assignment of the objective and expansion of weighted likelihood of the goal is done so that it can be localized in an image sequence. The role of the weight is significant as it allows functioning of optimization, which is gradient, based to be performed, which would not be practicable in a context of standard likelihood representations. The object that is to be tracked and elements belonging to local backgroundboth are modeled by the algorithm and scale changes in objective's appearance are handled. Accurate and increased objective localization is provided in non trivial scenarios (e.g. shadows) by this algorithm .

\subsection{Recognition of Gaussian Mixture Model using}

\section{Mean Variance Mapping Optimization[4]}

This approach allows fair representation of variouskinds of load distributions as a convex combination of variousnormal distributions with respective means and standard deviation. Various mixture mechanisms (weight, mean, and standard deviation) are produced and that is expressed as a problem of identification and effective solution is providedby MVMO. MVMO approach effectively represents the load models .

\subsection{Optimization Approach using Graph: [5]}

Multimodal references are interpreted using graph matching optimization approach. This type of graph representation captures both salient entities and their inter-associations. Based on constraints modeled as links or nodes in these graphs, graph matching optimization process finds the best matching among two graphs. This type of structure and process is especially required for recognizing multimodal references.One graph can describe all the referring expressions and their inter-associations, and all the potential referents can be represented by the another graph.
The expansion of referent graph can be easily done to incorporate other modalities such as gaze inputs, although currently it corresponds to gesture input and conversation context. However once the size of the graph is increased graph matching can become intractable. If the size of the graphs is limited this approach is very effective.

\subsection{Optimality Theory}

Optimality Theory (OT) is model of language and grammar. Here, a set of well-formed constraints constitutes the grammar. To detect linguistic structures these constraints are applied simultaneously.

The idea of these constraints as soft, which means violable and conflicting is an innovation of Optimality Theory. Among the set of available interpretations, the best alternative (hence, optimal interpretation) is the one that arises for an utterance inside a particular context and it improves the degree of constraint satisfaction.

The following three mechanisms are the main principles of Optimality Theory: 1), For each input,generator creates a group of possible outputs if a group of input is given. 2)Assessor selects the optimal output for that input, from the group of aspirant output. 3) In term of the ranking of constraints there is a firm dominance. Ranking of the constraints and constraints are fixed. Main principles of Optimality Theory can be applied to other representations even though it is grammar-based framework for natural language processing, Optimization for multimodal interpretation is addressed by graph-based approach; in particular, interpreting multimodal references. This method achieves the best interpretation among all alternatives as temporal, semantic, and contextual constraints together are simultaneously applied.

\section{HYBRID METHODS OF OPTIMIZATION}

Hybridization is an effective approachas it merges the best features of several algorithms. Hybridization is simply the fusion of two or more techniques so that their performances can be increased by the use of their good properties together.

To improve the algorithms that are currently in existence so that they can suit particular applicationsresearch is being done. Two approaches are used for improvement: (a) In first one the current algorithm is adapted or (b) by hybridizing the algorithms that are currently in existence. Another way of improvement is to fuse the assets or strong features of several algorithms.

\section{CONCLUSION}

In this paper need of optimization and techniques specially used for optimization in gesture recognition are discussed. It is concluded that GMM approach has advantage that different kinds of load distributions can be completely represented as a convex combination of several standard distributions with respective means and standard deviation 
Thus the conclusion is that hybrid optimization methods provides an efficient solution in the problems of optimized fusion of gestures.

\section{REFERENCES}

[1] Zhiyuan Weng and Petar M. Djuric "Data Fusion Based On Convex Optimization",ICASSP, 978-14799-0356-6/13/\$31.002013 IEEE.

[2] Vasileios Karavasilis , Christophoros Nikou and Aristidis Likas, "Visual Tracking by Weighted Likelihood Maximization", 2012 IEEE 24th International Conference on Tools with Artificial Intelligence.

[3] F. M. Gonzalez-Longatt, J. L. Rueda , I. Erlich , D. Bogdanov , W. Villa"Identification of Gaussian Mixture Model using Mean Variance Mapping Optimization: Venezuelan Case" , 2012 3rd IEEE PES Innovative Smart Grid Technologies Europe (ISGT Europe), Berlin.

[4] Francisco Gonzalez-Longatt, José Rueda, István Erlich, Dimitar Bogdanov,"Mean Variance Mapping Optimization for the Identification of Gaussian Mixture Model: Test Case.

[5] Joyce Y. Chai, Pengyu Hong+,Michelle, X. ZhoułZahar Prasov, "Optimization in Multimodal Interpretation". 